\title{
Oryctini (Coleoptera: Scarabaeidae: Dynastinae) da coleção de invertebrados do Instituto Nacional de Pesquisas da Amazônia (INPA), Manaus, Amazonas, Brasil.
}

Hector Jaime GASCA ${ }^{1}$, Claudio Ruy Vasconselos da FONSECA²

RESUMO

A Coleção de Invertebrados do Instituto Nacional de Pesquisas da Amazônia (INPA) possui 554 espécimes de Oryctini (Coleoptera: Scarabaeidae: Dynastinae), pertencentes a 7 gêneros, 16 espécies e 2 subespécies. As distribuições geográficas das espécies são fornecidas, sendo que $97 \%$ do material examinado procedem de coletas feitas em diferentes locais da Amazônia brasileira.

PALAVRAS-CHAVE: Scarabaeidae, Oryctini, Coleções, Distribuição geográfica, Amazônia brasileira.

\section{Oryctini (Coleoptera: Scarabaeidae: Dynastinae) of the invertebrate collection of the National Institute of Research of Amazonia (INPA), Manaus, Amazonas, Brazil.}

\section{ABSTRACT}

The Invertebrate Collection of the Instituto Nacional de Pesquisas da Amazônia (INPA), Manaus, Amazonas, Brazil, has 554 specimens of Oryctini (Coleoptera: Scarabaeidae: Dynastinae), belonging to seven genera, 16 species and two subspecies. Information about geographical distribution are provided, of which $97 \%$ of the material examined was collected from several places in the Brazilian Amazon.

KEY WORDS: Scarabaeidae, Oryctini, Collections, Geographical distribution, Brazilian Amazon.

1 Instituto Nacional de Pesquisas da Amazônia - INPA. Coordenação de Pesquisas em Entomologia. Av. André Araújo, 2936 - Petrópolis. CEP 69011-970 Manaus, Amazonas, Brasil. e-mail: hector@inpa.gov.br. Endereço atual: Corporación Sentido Natural. Calle 134 A \# 14 44. Bogotá, Colômbia. e-mail: hjgasca@sentidonatural.org.

2 Instituto Nacional de Pesquisas da Amazônia - INPA. Coordenação de Pesquisas em Entomologia. Av. André Araújo, 2936 - Petrópolis. CEP 69011 -970 Manaus, Amazonas, Brasil. e-mail: rclaudio@inpa.gov.br 
A tribo Oryctini (Scarabaeidae: Dynastinae) é uma das mais diversas devido ao número de espécies, a sua distribuição mundial, especialmente pantropical, e pela considerável situação de áreas de endemismo na América Central e do Sul (Mizinuma, 1999). Compreende 26 gêneros e 230 espécies no mundo, sendo que mais da metade dos gêneros (14) e espécies (135) ocorrem na região Neotropical (Ratcliffe e Cave, 2006). No Brasil ocorrem 7 gêneros e 44 espécies, constituindo 20\% da diversidade neotropical. Até o momento são conhecidas 46 espécies para a região Afrotropical e 31 espécies para a região Oriental (Endrödi, 1985).

Os orictínios adultos são ativos durante a noite e são atraídos freqüentemente pelas fontes luminosas com lâmpadas. Durante o dia procuram refúgio e permanecem escondidos. Algumas espécies se alimentam de matéria vegetal em decomposição ou de frutos doces apodrecidos, enquanto outras perfuram os caules das plantas como cana-de-açúcar e diferentes espécies de palmeiras (Ratcliffe, 2003). As larvas se desenvolvem no solo, em acumulações de resíduos vegetais, dentro dos caules de plantas, em formigueiros ou dentro de troncos e raízes grandes apodrecidas. Seu ciclo de vida completo pode demorar até dois anos (Morón et al., 1997). Algumas espécies são pragas de plantas de interesse econômico, atacando raízes e caules de várias espécies de palmeiras (Bodkin, 1919; Cockerell, 1946; Gonçalves, 1946; Vayssiere, 1965), de folhas de agaváceas (Nevermann, 1933) e de canade-açúcar (Morón et al., 1997; Ratcliffe, 2003).

A Coleção de Invertebrados do INPA é uma das mais recentes do Brasil com menos de 30 anos de desenvolmimento (Henriques, 1995), mas possui uma importante representação de espécies de insetos, principalmente da região Amazônica. A coleção de Coleoptera contém aproximadamente 2.000.000 de exemplares, dos quais cerca de 25.000 estão montados e 15.000 identificados até o nível de família, sendo que 75 gêneros e 198 espécies pertencem à família Scarabaeidae (Costa et al., 2000).

Foram estudados os exemplares secos alfinetados cujas etiquetas continham pelo menos a informação do local de coleta. A identificação dos espécimes foi feita com base nas descriçôes e chaves para gêneros e espécies de Oryctini (Endrödi, 1985; Ratcliffe, 2003; Ratcliffe e Cave, 2006), e por comparação com material previamente identificado da coleção do INPA. A classificação e nomenclatura utililizada seguiu Endrödi $(1976,1985)$.

A Coleção de Invertebrados do INPA contem 554 indivíduos pertencentes a 7 gêneros e 16 espécies e 2 subespécies de Oryctini (Tabela 1), com uma relação de sexos de cerca de 1:1 (51,44\% machos; 48,56\% fêmeas). A revisão do material de orictínios permitiu ampliar o acervo da coleção de referência em 130 exemplares, os quais se encontravam depositados na miscelânea de Coleoptera. A maioria dos exemplares é proveniente de coletas feitas com armadilha de luz mista de mercúrio, ou de luz ultravioleta do tipo BL (blacklight) e do tipo BLB (black-light blue), durante expediçôes realizadas no período de 1979 a 2000.

O material examinado foi coletado quase exclusivamente na Amazônia brasileira (97\%), ao longo do rio Amazonas e nas proximidades das principais cidades localizadas nas suas margens. O Estado melhor representado foi o Amazonas, com 11 espécies e 2 subespécies, representando $84 \%$ do acervo de Oryctini na coleção. Apenas três espécimes procedem de outros Estados do Brasil, enquanto 15 foram coletados fora do país: seis de Strategus aloeus (L.), um de Enema endymion Chevrolat, seis de Xyloryctes jamaicensis (Drury, 1773) do México, e dois de Xyloryctes sp do Panamá (Tabela 1).

As espécies melhor representadas foram Enema pan (Fabricius) (40\%) e Strategus aloeus (L.) (30\%). A Coleção de Invertebrados do INPA possui $36,3 \%$ da riqueza de Oryctini da fauna brasileira (44 espécies). Estes resultados, junto com os dados de ocorrência das espécies de Oryctini na Amazônia brasileira, indicam a necessidade de realizar um maior esforço de coleta em Estados pouco explorados como Maranhão e Tocantins, com o objetivo de obter uma melhor representatividade da fauna da região e de complementar as informaçōes sobre a distribuição geográfica e história natural deste grupo de besouros. 


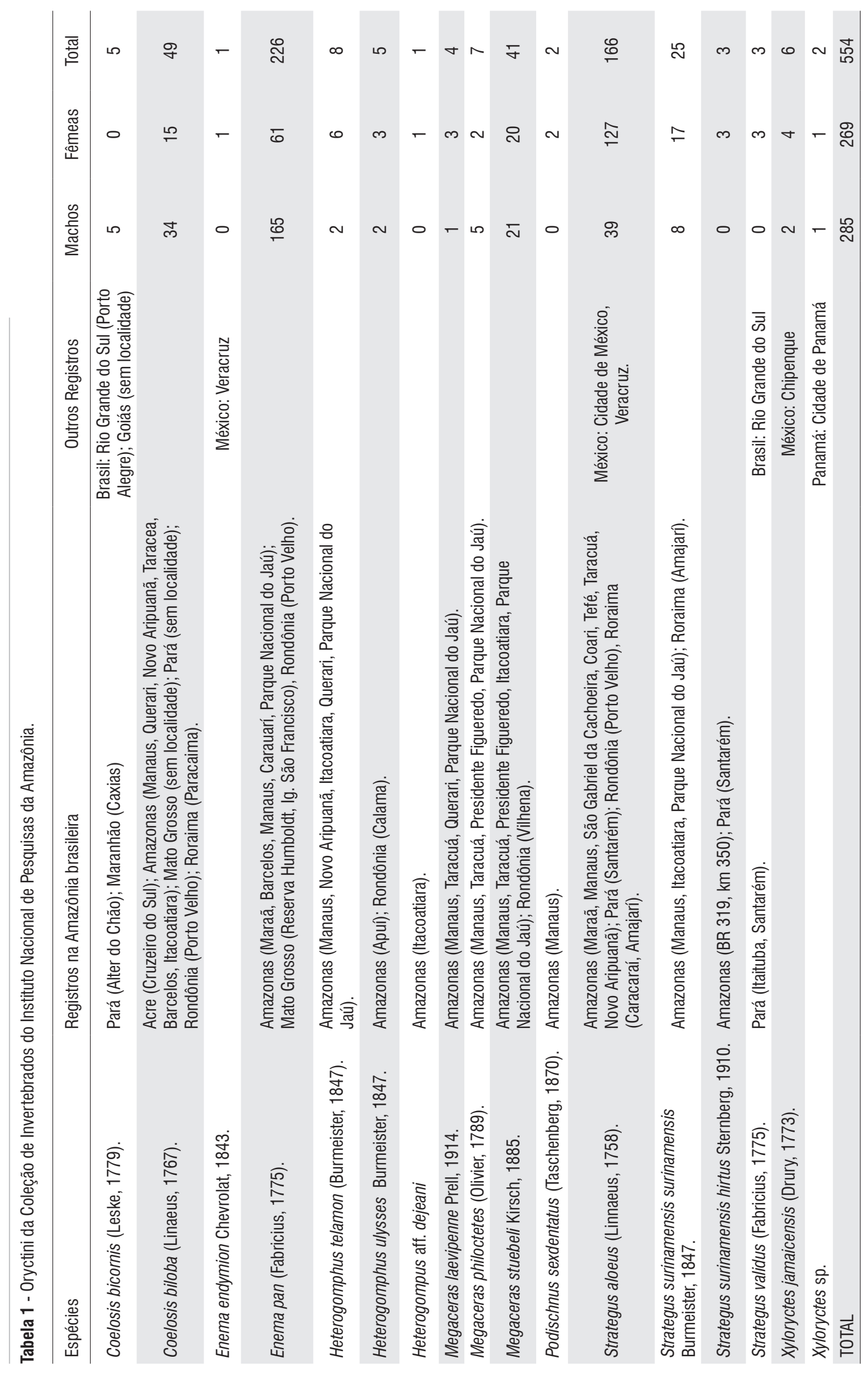




\section{AGRADECIMENTOS}

Ao Dr. Augusto Loureiro Henriques, curador da Coleção de Invertebrados do INPA, por facilitar o acesso ao material depositado na coleção do INPA. Ao Dr. Brett C. Ratcliffe, do Museu Estadual da Universidade de Nebraska, EUA, pela identificação e confirmação de algumas espécies de Oryctini. Ao Dr. Márcio Luís Leitão Barbosa, do INPA, pelos valiosos comentários e sugestôes ao manuscrito.

\section{BIBLIOGRAFIA CITADA}

Bodkin, G.E. 1919. Notes on the Coleoptera of British Guiana. Entomologists Monthly Magazine, 55: 210-219.

Cockerell, T.D. 1946. Strategus injuring date-palms. Entomological News, 17: 34.

Costa, C.; Ide, S.; Rosado-Neto, G.H.; Galileo, M.H.M.; Fonseca, C.R.V.; Valente, R. M.; Monné, M.A. 2000. Diagnóstico del conocimiento de las principales coleciones brasilenãs de Coleoptera. In: Martín-Piera, F.; Morrone, J. J.; Melic, A. (Eds.). Hacia un proyecto Cyted para el inventario y estimación de la diversidad entomológica en Iberoamérica: PrIBES-2000. Zaragoza, Vol. 1. Sociedad Entomológica Aragonesa SEA. p. 115-136.

Gonçalves, C.R. 1946. Males de carnaúba no Ceará e no Piaui. Boletim Fitossanitário, 3: 145-170.

Endrödi, S. 1976. Monographie der Dynastinae 5.Tribus: Oryctini (die Arten von Amerika) (Coleoptera: Melolonthinae). Folia Entomologica Hungarica, 29: 9-174.

Endrödi, S. 1985. The Dynastinae of the World. Dr W. Junk. Publ. Dordrecht, Netherlands. 800pp.
Henriques, A.L. 1995. A coleção de Tabanidae (Insecta: Diptera) do Instituto Nacional de Pesquisas da Amazônia (INPA), Amazonas, Brasil. Boletim Do Museu Paraense Emilio Goeldi, série Zoológica, 11(1): 57-99.

Morón, M.A.; Ratcliffe, B.C.; Deloya, C. 1997. Atlas de los escarabajos de México (Coleoptera: Lamellicornia) Vol 1. Comisión Nacional para el Conocimiento y Uso de la Biodiversidad (CONABIO) y Sociedad Mexicana de Entomologia. México. 280 pp.

Mizinuma. T. 1999. Giant Beetles. Euchirinae-Dynastinae. Endless Collection Series. Vol 3. Endless Science Information. Tokyo, Japan. 122 pp.

Neverman, F. 1933. Beobachtungen über die Lebensweise einiger Lamellicornier und einer Chrysomelidae. Entomologische Blätter, 29: 179-183.

Ratcliffe, B.C. 2003. The Dynastinae Scarab Beetles of Costa Rica and Panamá. Bulletin of the University of Nebraska State Museum 16: $1-506$.

Ratcliffe, B.C.; Cave, R. 2006. The Dynastinae Scarab Beetles of Honduras, Nicaragua and El Salvador. Bulletin of the University of Nebraska State Museum 21: 1-424.

Vayssiere, P. 1965. Sur quelques insects des palmiers en Amérique de Sud. Mededel Landouwhogesch Opzoekingssta Ghent, 30: 1571-1576.

Recebido em 03/11/2008

Aceito em 02/01/2009 